Article

\title{
From Crystal Structures of RgIA4 in Complex with Ac-AChBP to Molecular Determinants of Its High Potency of $\alpha 9 \alpha 10$ nAChR
}

\author{
Si Pan ${ }^{1,+}\left(\mathbb{D}\right.$, Yingxu Fan ${ }^{2,+}$, Xiaopeng Zhu ${ }^{3} \mathbb{C}$, Yi Xue $^{2, *}$, Sulan Luo ${ }^{3,4, * \mathbb{1}}$ and Xinquan Wang ${ }^{5, *}$ \\ 1 Key Laboratory of Translational Tumor Medicine in Fujian Province, Putian University, Putian 351100, China; \\ plar000@163.com \\ 2 Tsinghua-Peking Joint Center for Life Sciences, School of Life Sciences, Tsinghua University, \\ Beijing 100084, China; fanyx17@mails.tsinghua.edu.cn \\ 3 Medical School, Guangxi University, Nanning 530004, China; biozxp@163.com \\ 4 Key Laboratory of Tropical Biological Resources of Ministry of Education, Key Laboratory for Marine Drugs \\ of Haikou, School of Life and Pharmaceutical Sciences, Hainan University, Haikou 570228, China \\ 5 Beijing Advanced Innovation Center for Structural Biology, Collaborative Innovation Center for Biotherapy, \\ The Ministry of Education Key Laboratory of Protein Science, School of Life Sciences, Tsinghua University, \\ Beijing 100084, China \\ * Correspondence: yixue@mail.tsinghua.edu.cn (Y.X.); luosulan2003@163.com (S.L.); \\ xinquanwang@mail.tsinghua.edu.cn (X.W.) \\ + These authors contributed equally to this work.
}

Citation: Pan, S.; Fan, Y.; Zhu, X.; Xue, Y.; Luo, S.; Wang, X. From Crystal Structures of RgIA4 in Complex with Ac-AChBP to Molecular Determinants of Its High Potency of $\alpha 9 \alpha 10$ nAChR. Mar. Drugs 2021, 19, 709. https:// doi.org/10.3390/md19120709

Academic Editor: Bill J. Baker

Received: 5 November 2021

Accepted: 14 December 2021

Published: 17 December 2021

Publisher's Note: MDPI stays neutral with regard to jurisdictional claims in published maps and institutional affiliations.

Copyright: (c) 2021 by the authors. Licensee MDPI, Basel, Switzerland. This article is an open access article distributed under the terms and conditions of the Creative Commons Attribution (CC BY) license (https:/ / creativecommons.org/licenses/by/ $4.0 /)$.
Abstract: $\alpha 9$-containing nicotinic acetylcholine receptors (nAChRs) have been shown to play critical roles in neuropathic pain. The $\alpha$-conotoxin ( $\alpha$-CTx) RgIA and its analog RgIA4 were identified as the most selective inhibitor of $\alpha 9 \alpha 10 \mathrm{nAChR}$. However, the mechanism of their selectivity toward $\alpha 9 \alpha 10$ nAChR remains elusive. Here, we reported the co-crystal structure of RgIA and RgIA4 in complex with Aplysia californica acetylcholine binding protein (Ac-AChBP) at resolution of $2.6 \AA$, respectively. Based on the structure of the complexes, together with molecular dynamic simulation (MD-simulation), we suggested the key residues of $\alpha 9 \alpha 10 \mathrm{nAChR}$ in determining its high affinity for RgIA/RgIA4. This is the first time the complex between pain-related conotoxins and Ac-AChBP was reported and the complementary side of $\alpha 9$ subunit in binding of the antagonists shown. These results provide realistic template for the design of new therapeutic in neuropathic pain.

Keywords: acetylcholine binding protein; nicotinic acetylcholine receptors; $\alpha$-conotoxin; $\operatorname{RgIA}$; RgIA4; crystal structure; molecular dynamics simulation

\section{Introduction}

Nicotinic acetylcholine receptors (nAChRs) are the best characterized members of the pentameric ligand-gated ion channels (pLGICs) family that also includes receptors for serotonin (5-HT3), GABA A $_{\text {, }}$ inc-activated channels (ZAC receptor), and glycine receptors $[1,2]$. They can be further divided into two types: the neuronal type nAChRs and muscle-type nAChRs. Neuronal nAChRs are mainly located in the central and peripheral nervous systems and mediate fast neurotransmission [3]. In vertebrates, neuronal $\mathrm{nAChRs}$ are composed of a combination of eight $\alpha(\alpha 2-\alpha 7, \alpha 9-\alpha 10)$ and three $\beta(\beta 2-\beta 4)$ subunits, forming either homopentamers or heteropentamers (e.g., $\alpha 7, \alpha 4 \beta 2, \alpha 3 \beta 2$, and $\alpha 9 \alpha 10$ nAChRs) [4]. All $\alpha$ and $\beta$ subunits share a similar architecture with an $\mathrm{N}$-terminal extracellular ligand-binding domain, a transmembrane domain consisting of four $\alpha$-helixes, and a short C-terminal extracellular tail. The binding of neurotransmitters acetylcholine to the extracellular domain induces structural rearrangements of the transmembrane domain, leading to a rapid opening of the central ion-conducting pore in the pentameric $\mathrm{nAChRs}$ [5].

Neuronal nAChRs are implicated in nicotine addiction, abuse of drugs, and various neurological and non-neurological diseases such as neuropathic pain, Alzheimer's, Parkin- 
son's diseases, and inflammation [6]. Therefore, it is highly desirable to develop nAChR antagonists with high potency and selectivity as therapeutic agents and pharmacological tools. $\alpha$-conotoxins ( $\alpha$-CTxs) from Conus genus are a family of small, disulfide-linked peptide neurotoxins that are competitive antagonists of nAChRs with unparalleled potency and selectivity [7]. Classical $\alpha$-conotoxins are generally 12-20 amino acids in length with four cysteine residues in a framework of CC-C-C. Typically, $\alpha$-conotoxin globular isomer with CysI-CysIII and CysII-CysIV connectivity and $\alpha$-helical backbone is the naturally occurring bioactive form. Several $\alpha$-conotoxins showing selectivity for different nAChR subtypes have been identified as potent antagonists of chronic pain, such as Vc1.1 and RgIA. Early publications suggested that Vc1.1 could selectively inhibit rat $\alpha 9 \alpha 10 \mathrm{nAChR}$ with the affinity of $19 \mathrm{nM}$. Unfortunately, a clinical trial of Vc1.1 was dropped during phase IIa since its potency at human $\mathrm{nAChR}$ was 100 -fold lower than that at rat $\mathrm{nAChR}$ [8]. Subsequently, Vc1.1 was reported to inhibit human dorsal root ganglion neuron via activating the $G_{A B A}$ receptor [9]. Up to now, it is still unclear whether the inhibition of $\alpha 9 \alpha 10 \mathrm{nAChR}$ or activation of $\mathrm{GABA}_{\mathrm{B}}$ receptor is the analgesic mechanism. Our present study focused on $\alpha$-CTx RgIA and its targeting receptor $\alpha 9 \alpha 10 \mathrm{nAChR}$. It was found that $\alpha 9$-containing $\mathrm{nAChRs}$ including $\alpha 9 \alpha 10$ play critical roles in neuropathic pain after chemotherapy-induced neuropathy and traumatic injury to nerves [10]. The $\alpha 9$ and $\alpha 10$ subunits share a high sequence similarity, especially in the extracellular domain (ECD) region with $77 \%$ identity [11]. In contrast to the $\alpha 9$ subunit, $\alpha 10$ subunits do not form functional receptors by themselves but do assemble with $\alpha 9$ subunits to form $\alpha 9 \alpha 10$ heteropentamer [12]. RgIA, a 13-amino-acid $\alpha$-conotoxin isolated from the carnivorous marine snail, is a highly selective $\alpha 9 \alpha 10 \mathrm{nAChR}$ antagonist, showing $>1000$-fold higher potency in inhibiting $\alpha 9 \alpha 10$ than other $\mathrm{nAChRs}$. RgIA blocks rodent $\alpha 9 \alpha 10 \mathrm{nAChR}$ with high potency and has been shown to be effective in rodent models of neuropathic pain [13]. However, it is approximately 300-fold less active on human $\alpha 9 \alpha 10 \mathrm{nAChR}$ due to a single-site amino acid substitution in human $\alpha 9$ subunit, which limits potential therapeutic application of RgIA [14]. RgIA4, an engineered analog, was reported to exhibit high potency on both human and rodent $\alpha 9 \alpha 10 \mathrm{nAChR}$, and has been shown to effectively prevent oxliplatin-induced pain in rats and mice $[15,16]$. Therefore, the RgIA4/ $\alpha 9 \alpha 10$ pair is a critical and promising target for developing and optimizing therapeutic agents for prevention of chronic neuropathic pain.

Structural information of the ligand-binding site in the pentameric $\alpha 9 \alpha 10$ receptor and its complex with RgIA would greatly advance our understanding of the molecular basis for the high potency of RgIA against the $\alpha 9 \alpha 10 \mathrm{nAChR}$. The $\alpha$-CTx-binding site in the pentameric nAChR usually consists of the principal (+) side of the ECD of an $\alpha$ subunit and the complementary (-) side of the ECD of the adjacent $\alpha$ or $\beta$ subunit. Recently, the crystal structure of monomeric extracellular domain of $\alpha 9$ subunit bound with RgIA was determined [17]. However, this structure only displayed the principal side between $\alpha 9$-ECD and RgIA, and the complementary side of $\alpha 10-\mathrm{ECD}$ and the interaction with RgIA is still unknown. Therefore, we have not fully understood how RgIA achieved high inhibitory activity against the rodent $\alpha 9 \alpha 10$ receptor, as well as how the modification in RgIA4 closes the affinity gap across the rodent and human receptors.

Currently, a few pentameric $\mathrm{nAChR}$ receptor structures including Torpedo $\mathrm{nAChR}$, $\alpha 7 \mathrm{nAChR}$ chimera, $\alpha 4 \beta 2 \mathrm{nAChR}$, and human $\alpha 2 \mathrm{ECD}$ pentamer have been determined by $\mathrm{X}$-ray diffraction and cyro-EM methods, possibly due to the difficulties encountered in expressing and assembling the pentameric receptors [18-21]. One significant progress in the structural and functional studies of nAChRs was the discovery and crystallization of acetylcholine-binding protein (AChBP), which is a soluble homopentamer and firstly isolated from the snail Lymnaea stagnalis [22,23]. Sequence alignment showed that AChBPs are most closely related to the ECDs of nAChRs, especially the $\alpha$-subunits in nAChRs, and nearly all conserved residues in nAChRs for ligand binding are present in AChBP [22]. Thus, pentameric AChBP is an excellent structural and functional homolog of the ECDs of most nAChRs. The first isolated and crystalized AChBP was from Lymnaea stagnalis (Ls-AChBP), but most crystal structures of different $\alpha$-CTxs have been solved for the 
complexes with the AChBP from Aplysia californica (Ac-AChBP) and served as important models for studying $\alpha-C T x / n A C h R$ interactions at the atomic level.

Here, we reported the co-crystal structures of Ac-AChBP bound with RgIA and RgIA4, respectively. We analyzed the structural interfaces in these two complexes and revealed detailed interactions of RgIA and RgIA4 with $A c$-AChBP, respectively. Based on the determined complex structures of $\alpha$-CTxs with Ac-AChBP, we also built the complexes of $\alpha 9 \alpha 10$ $\mathrm{nAChR}$ with RgIA and RgIA4 by homologous modeling and MD-simulation. By analyzing these two $\alpha$-CTx / $\alpha 9 \alpha 10$ complexes, we also revealed the molecular basis underlying how the modifications of two residues in RgIA4 result in additional interactions and increase the affinity with human $\alpha 9 \alpha 10 \mathrm{nAChR}$. Since the $\alpha 9 \alpha 10 \mathrm{nAChR}$ is an important pharmacotherapeutic target for the pain model, our study provides significant implications for the design of highly selective therapeutic $\alpha$-conotoxin analogs for use against nAChR-related diseases.

\section{Results}

\subsection{Overall Structure of RgIA and RgIA4 Bound to Ac-AChBP}

To investigate the binding at atomic details, we determined the complex structures of Ac-AChBP bound with RgIA and RgIA4, respectively. The amino acid sequence of RgIA is GCCSDPRCRYRCR-, and the folded peptide has two disulfide bonds connecting Cys2 to Cys8 and Cys3 to Cys12. The RgIA4 was derived from RgIA by substituting Arg9 and Tyr10 with Citrulline (represented as CIR) and 3-Iodo-Tyrosine (represented as IYR), respectively. The RP-HPLC and electrospray ionization mass spectrometry (ESIMS) were utilized to purify and characterize the synthesized and refolded RgIA and RgIA4 (Figure S1). The Ac-AChBP was expressed in Hi5 insect cells and purified by gelfiltration column. Both complex structures, RgIA/Ac-AChBP and RgIA4/Ac-AChBP, were determined by X-ray crystallography at $2.6 \AA$ resolution (Figure 1, Table S1). In these structures, five monomers of $A c-\mathrm{AChBP}$ assemble into a homopentamer, resembling a windmill toy with petal-like monomers. The overall pentameric structure is analogous to the quaternary structures of nAChR ECDs (Figure 1). As shown in the Figure 1, the Ac-AChBP homopentamer has five ligand binding sites, each located in a cleft between two adjacent subunits (Figure 1B,D). All five ligand-binging sites are occupied by the RgIA in the RgIA / Ac-AChBP complex (Figure 1A), and only three RgIA4 peptides were found in the RgIA4/Ac-AChBP complex (Figure 1C). The loops A, B, and C of one Ac-AChBP monomer are involved on the ligand binding at the principal side and the loops $\mathrm{D}, \mathrm{E}$, and $\mathrm{F}$ and several $\beta$-strands of another monomer are involved in the ligand binding at the complementary side (Figure 2D). In the ligand-binding site, the RgIA and RgIA4 overall structures were very similar with a central short helix and unstructured $\mathrm{N}$ - and C-terminus (Figure 2A,B). Structural comparison showed that the RgIA in the complex and unbound RgIA have obvious backbone conformational differences, indicating the binding with Ac-AChBP could probably induce conformational changes in RgIA (Figure 2C).

\subsection{Interactions of RgIA with Ac-AChBP}

To fully reveal the interactions between RgIA and $A c-A C h B P$, we performed a structural analysis of the principal and complementary sides in the RgIA/Ac-AChBP complex (Figure $3 \mathrm{~A}$ and Table 1). A mixture of hydrophobic and hydrophilic interactions occurred on the principal interaction side, and the Ac-AChBP-contacting residues were mainly from the loop B (Trp145) and loop C (Gln184-Tyr193) (Figure 3A and Table 1). Like other structures of typical $\alpha$-CTxs bound to Ac-AChBP, the Cys2-Cys 8 disulfide bridge of the RgIA stacked onto the vicinal Cys188-Cys189 disulfide bond of the Ac-AChBP. Hydrogen bonding interactions were formed between Asp5, Arg7, and Tyr10 of RgIA and Gln184, Tyr186, and Glu191 from loop C of the Ac-AChBP. Additionally, Glu191 of Ac-AChBP formed a salt bridge interaction with Arg11 of RgIA. Other interactions included the contacts of Pro6 and Arg7 of RgIA with Trp145 (loop B) and Tyr91 (loop A) of Ac-AChBP, respectively. The complementary interaction side was formed by $\beta$-strands, loop D (Gln55, ArG57), loop E (Met114), and loop F (Asp162, Ser164, and Ser165) of adjacent Ac-AChBP (Figure 3B 
and Table 1). On this side, a significant interacting residue of RgIA was Ser4, whose side chain resided in a pocket contacting with Asp162 (loop F), Ser164 (loop F), and Ser165 of Ac-AChBP through several hydrogen bonds. Another hydrogen bond was formed between Asp75 of Ac-AChBP and Tyr10 of RgIA. Besides, a salt bridge interaction was formed between Arg57 (loop D) of Ac-AChBP and Arg13 of RgIA.

A

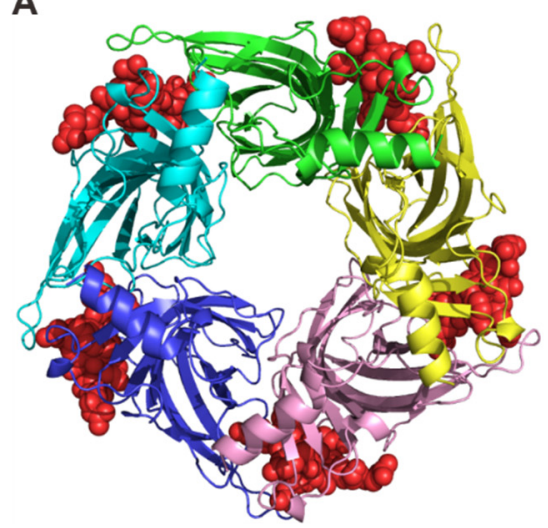

C

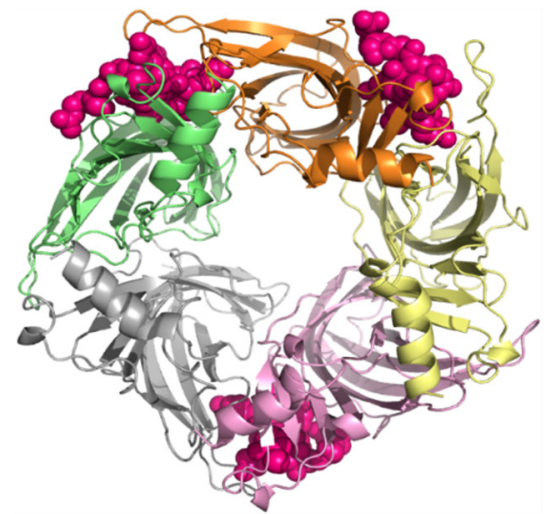

B

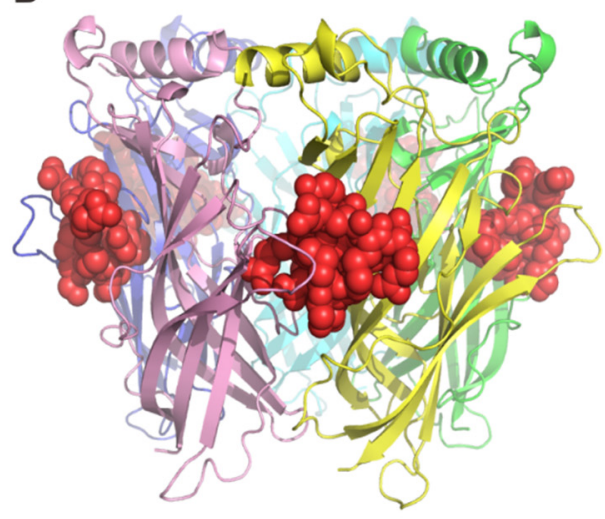

D

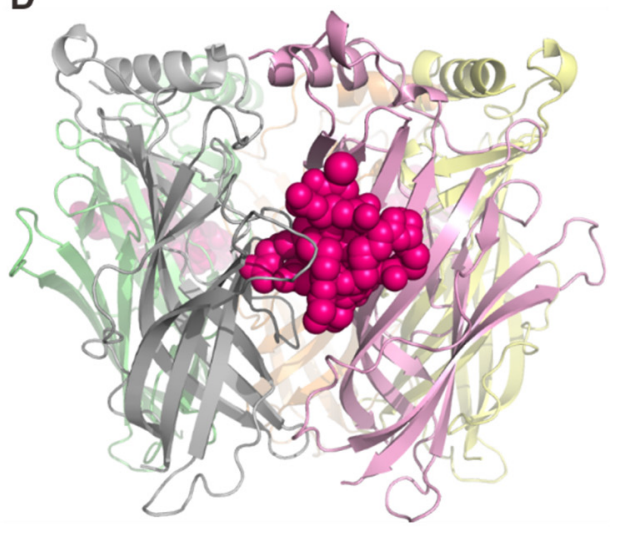

Figure 1. The X-ray crystal structure of $A c$-AChBP in complex with RgIA and RgIA4, respectively. The PDB IDs of these complexes are 7EGR (RgIA-Ac-AChBP) and 7EGX (RgIA4-Ac-AChBP). (A) The top view of the pentameric structure with five Ac-AChBP protomers, each in different colors and five $\alpha$-CTx RgIA molecules in red. (B) The side view of the pentamer with RgIA molecules (in red). (C) The top view of the pentameric structure with five Ac-AChBP protomers, each in different colors and with RgIA analog RgIA4 molecules in magenta. (D) The side view of the pentamer with RgIA molecules (in magenta).

\subsection{Interactions of RgIA4 with $A c-A C h B P$}

Unlike RgIA showing 300 -fold higher potency on the rodent $\alpha 9 \alpha 10 \mathrm{nAChR}$, the analog RgIA4 with Arg9 and Tyr10 replaced by CIR and IYR closed the affinity gap across the rodent and human $\alpha 9 \alpha 10 \mathrm{nAChR}$. To provide insights into the underlying molecular basis, we also determined the complex structure of RgIA4 with Ac-AChBP. Figure 3C,D show the structural features of the principal and complementary sides in the complex, respectively. On the principal side, Ac-AChBP residues Tyr91, Trp145, Tyr186, Cys188, Cys189, Glu191, and Tyr193 play key roles in binding RgIA4. Similar to other conotoxin/AcAChBP complexes, the Cys2-Cys8 disulfide bridge of the RgIA4 also stacked onto the vicinal Cys188-Cys189 disulfide bond of the Ac-AChBP. Different from RgIA/Ac-AChBP complex, the hydrogen bonding interactions involved Tyr193 (loop C) and Tyr91 (loop A) of the Ac-AChBP and Arg7 and Arg11 of RgIA4, respectively. Like the RgIA/Ac-AChBP complex, additional hydrophilic interactions such as the salt bridge also formed between Arg11 of RgIA4 and Glu191 (loop C) (Figure 3C and Table 1). On the complementary 
side, salt bridge interaction was also formed between Arg13 of RgIA4 and Arg57 (loop D) of the Ac-AChBP-like RgIA/Ac-AChBP complex. Residues CIR9 and IYR10 of the RgIA4 were involved in the interactions on the complementary side, where the contacting Ac-AChBP residues were mainly from the $\beta$-strands and loop D (Figure 3D). Different from RgIA/Ac-AChBP complex, on this side, hydrogen bonds included Ser4 (RgIA4) to Ser165 (Ac-AChBP), CIR9 (RgIA4) to Thr34 and Gln55 (Ac-AChBP), IYR10 (RgIA4) to Asp75 (Ac-AChBP), and Arg13 (RgIA4) to Gln55 (loop D of Ac-AChBP) (Figure 3D and Table 1).

A

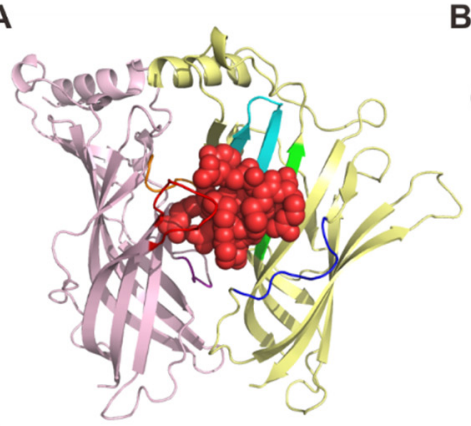

B

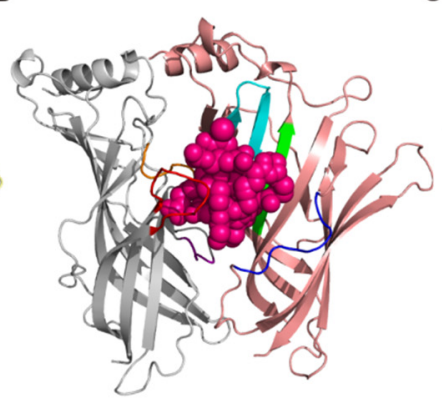

C

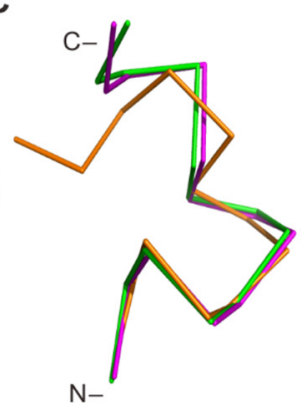

D

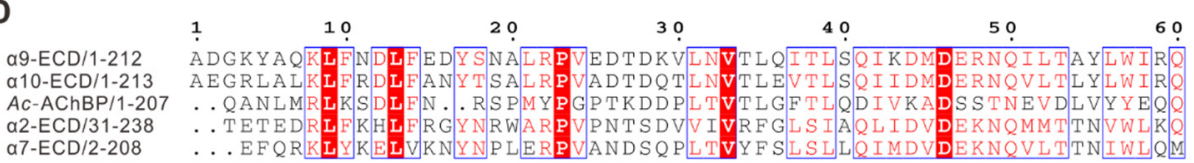

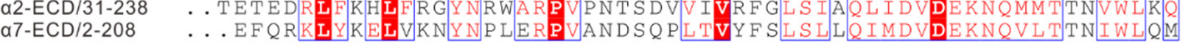
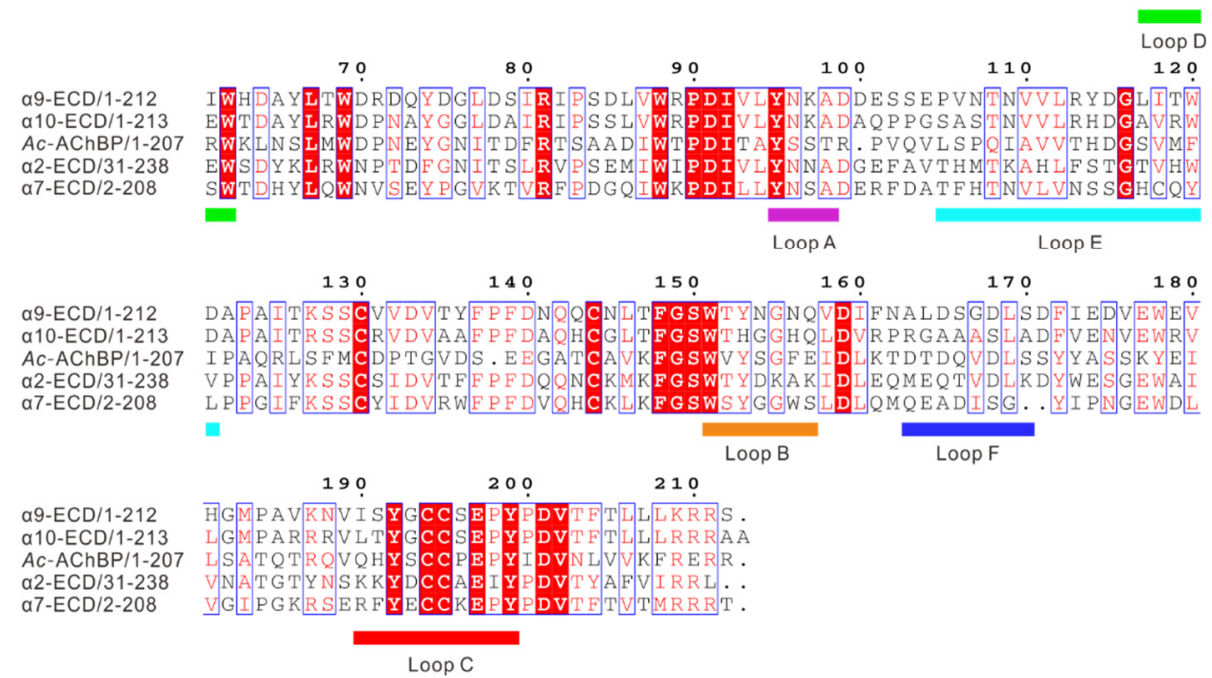

Figure 2. Schematic diagram of Ac-AChBP with RgIA/RgIA4 and homologous proteins. (A) The side view of two adjacent AChBP protomers of the pentamer with a bound RgIA molecule (in red). (B) The side view of two adjacent AChBP protomers of the pentamer with a bound RgIA4 molecule in magenta. (C) Superposition of RgIA/RgIA4 in different state. AChBP-bound RgIA in magenta, AChBP-bound RgIA4 in green, NMR structure of free-state RgIA in orange. Bottom, Multiple sequence alignment of RgIA, RgIA4, GIC, and LvIA. Disulfide bridges are shown in Cys1-Cya3 and Cys2-Cys4. (D) Sequence alignment of Ac-AChBP, human $\alpha 9, \alpha 10, \alpha 2$, and $\alpha 7$ nAChR ECD. 
A

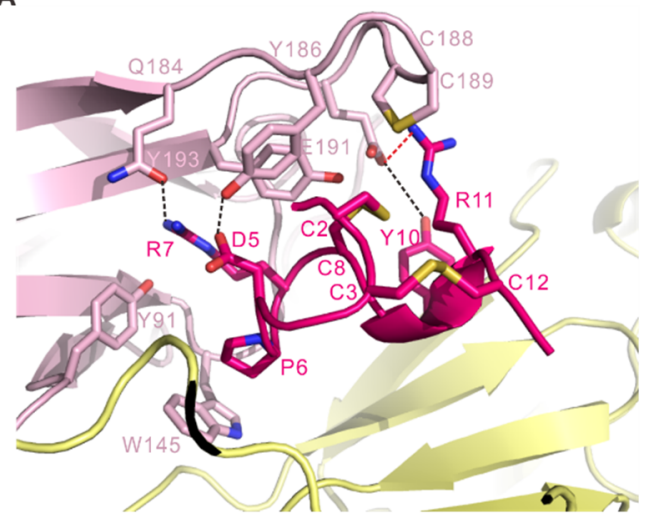

C

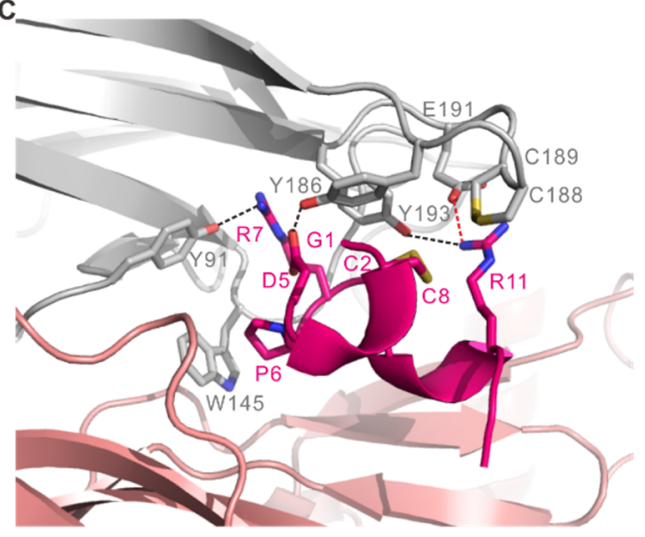

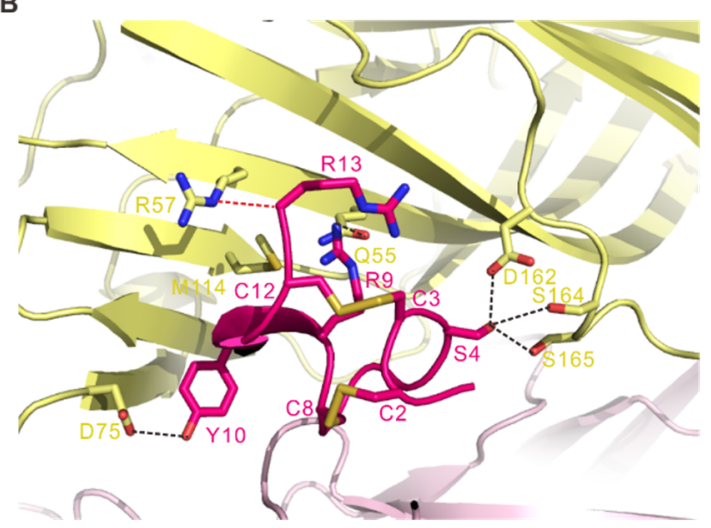

D

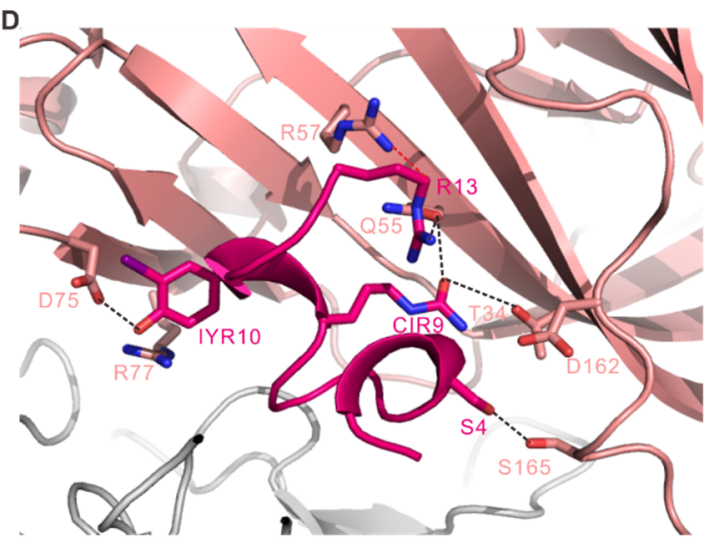

Figure 3. Ac-AChBP- $\alpha$-CTx binding interfaces. (A) The interactions on the principal side of RgIA-Ac-AChBP complex. Residues Asp5, Arg7, and Tyr10 of the RgIA form hydrogen bonds (represented by black dashed line) with Tyr186, Gln184, and Glu191 of Ac-AChBP, respectively. Salt bridge (represented by the red dashed line) is also observed between Arg11 of RgIA and Glu191 of Ac-AChBP. (B) The complementary side of the RgIA-Ac-AChBP complex. Hydrogen bonds are involved in Residues Ser4, Arg9, and Tyr10 of RgIA and Gln55, Asp75, Asp162, Ser164, and Ser165 of Ac-AChBP. Residue Arg13 of RgIA forms a salt bridge with Arg57 of Ac-AChBP. (C) The principal side of RgIA4-Ac-AChBP complex. Residues Asp5, Arg7, and Arg11 of the RgIA4 form hydrogen bonds with Tyr186, Tyr91, and Tyr193 of Ac-AChBP, respectively. Residue Arg11 of RgIA4 forms a salt bridge with Glu191 of Ac-AChBP. (D) The complementary side of RgIA4-Ac-AChBP complex. Hydrogen bonds are involved in Residues Ser4, CIR9, IYR10, and Arg13 of RgIA4 and Thr34, Gln55, Asp75, and Ser165 of Ac-AChBP. Residue Arg13 of RgIA4 forms a salt bridge with Arg57 of Ac-AChBP.

Table 1. Contacts between residues of $A c-A C h B P$ and $\operatorname{RgIA}$ and RgIA4, respectively.

\begin{tabular}{ccc}
\hline Ac-AChBP & RgIA & RgIA4 \\
\hline & Principal Side & \\
\hline Tyr91 & Arg7 & Arg7 \\
Trp145 & Pro6, Arg7 & Pro6 \\
Val146 & Arg7 & \\
Gln184 & Asp5, Arg7 & \\
Tyr186 & Asp5 & Gly1, Asp5 \\
Cys188 & & Cys2 \\
Cys189 & Cys8, Arg11 & Cys2, Cys8 \\
Glu191 & Tyr10, Arg11 & Arg11 \\
Tyr193 & Cys8 & Cys8, Arg11 \\
Ile194 & Arg7 & \\
\hline
\end{tabular}


Table 1. Cont.

\begin{tabular}{ccc}
\hline Ac-AChBP & RgIA & RgIA4 \\
\hline & Complementary Side & \\
\hline Thr34 & & CIR9 \\
Gln55 & Arg9 & CIR9, Arg13 \\
Arg57 & Arg13 & Arg13 \\
Asp75 & Tyr10 & IYR10 \\
Arg77 & & IYR10 \\
Met114 & Arg9 & \\
Asp162 & Ser4 & Ser4 \\
Ser164 & Ser4 & \\
Ser165 & Ser4 & Ser4 \\
\hline
\end{tabular}

\subsection{Homology Modelling of Human $\alpha 9 \alpha 10 n A C h R$ and Docking with RgIA and Its Mutant}

To gain further insight into the interactions of RgIA and RgIA4 with human $\alpha 9 \alpha 10$ nAChR, especially the increasing binding of RgIA4, we built the ECD pentamers of human $\alpha 9 \alpha 10$ nAChR with two possible stoichiometries $(\alpha 9)_{2}(\alpha 10)_{3}$ and $(\alpha 9)_{3}(\alpha 10)_{2}$ bound with RgIA and RgIA4 by homologous modeling, followed by MD-simulations. The models were constructed based on the X-ray crystal structures of the RgIA/Ac-AChBP and RgIA4/Ac-AChBP complexes. Subsequently, the generated models were validated in terms of stereochemical quality by Ramachandran plot (Figure S2). The results revealed that in over $90 \%$ of residues, two models were in the most favored and additional allowed regions, reflecting the good-quality models predicted and worth investigating further. There are three possible ligand binding sites in two different $\alpha 9 \alpha 10 \mathrm{nAChR}$ pentamers: $\alpha 9(+) / \alpha 10(-), \alpha 9(+) / \alpha 9(-)$, and $\alpha 10(+) / \alpha 9(-)$. Considering that interactions of RgIA with human $\alpha 9 \alpha 10 \mathrm{nAChR}$ have been modeled and described in previous studies, here we focused on the interaction of RgIA4 with receptor at the $\alpha 9(+) / \alpha 10(-), \alpha 9(+) / \alpha 9(-)$, and $\alpha 10(+) / \alpha 9(-)$ interface (Figure 4). These results about the interactions of RgIA with human $\alpha 9 \alpha 10$ nAChR are shown in Figure S3.

At the $\alpha 9(+) / \alpha 9(-)$ binding interface (Figure 4A,B), two $\alpha 9$ subunits served on the principal side and complementary side, respectively. As shown in Figure 4B, residues Tyr95, Trp151, Asn154, Glu197, Tyr199, and Pro200 of the $\alpha 9$ subunit are the key contacting residues on the principal side. Specific interactions included Tyr95 and Pro200 forming a hydrogen bond with Arg7 of RgIA4 and Glu197 interacts with Arg11 of RgIA4 through a salt bridge. On the complementary side, a notable interacting residue in the RgIA4 was Ser4, whose side chain resided in a pocket contacting with Thr38, Asp168, Ser170, and Asp171 of the other $\alpha 9$ subunit. Hydrogen bonds were formed between Ser4 of RgIA4 with Asp168, Ser170, and Asp171 of the $\alpha 9$ subunit, respectively. Near this interacting pocket, a salt bridge was formed between Gly1 of RgIA4 and Asp171 of the $\alpha 9$ subunit. Additionally, other hydrogen bonding interactions occurred between IYR10 of RgIA4 and Arg81 and Arg113 of the $\alpha 9$ subunit, respectively. Residues Thr38, Trp57, Arg59, and Asp121 of the $\alpha 9$ subunit had van der Waals contacts with CIR9 of RgIA4.

At the $\alpha 9(+) / \alpha 10(-)$ binding site (Figure $4 \mathrm{C}, \mathrm{D}$ ), the principal side exhibited subtle differences from that at the $\alpha 9(+) / \alpha 9(-)$ interface, although the contacting partners were the same between the $\alpha 9$ subunit and RgIA4. Two significant exceptions were Thr152 and Asp201 of the $\alpha 9$ subunit, which were not involved in the interaction at the principal side of $\alpha 9(+) / \alpha 9(-)$ binding interface. Thr152 and Asp201 of the $\alpha 9$ subunit interacted with IYR10 and Arg7 of RgIA4 through a hydrogen bond and salt bridge, respectively. Another exception is the hydrogen bond between Tyr192 of $\alpha 9$ subunit and Asp5 of RgIA4. On the complementary side, most interactions were similar to those of $\alpha 9(+) / \alpha 9(-)$ binding interface. It is obvious to show that there were two binding pockets around Ser4 and Arg13 of RgIA4. The side chain of Ser4 was located in the pocket which is composed of Thr38, Ser168, and Asp171 of the $\alpha 10$ subunit. Additionally, Ser4 of RgIA4 formed hydrogen bonds with Thr38 and Asp171, respectively. Arg13 of RgIA4 interacted with Arg59, Arg119 
and Arg163 through salt bridges. Compared with the $\alpha 9(+) / \alpha 9(-)$ binding interface, there was an additional hydrogen bond between Arg81 and Arg113 of the $\alpha 10$ subunit and IYR10 of RgIA4 (Figure 4D).
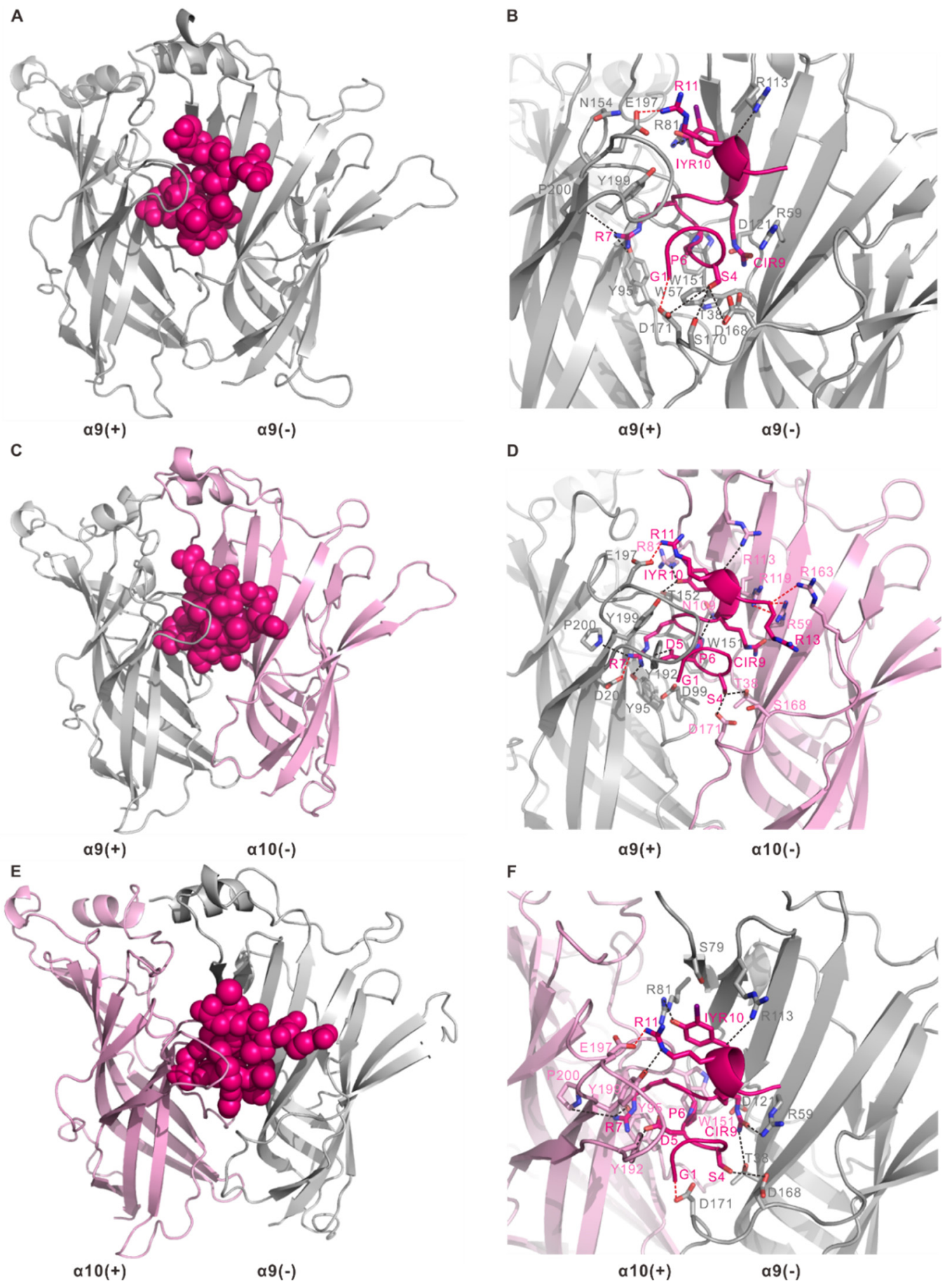

Figure 4. Molecular dynamics models of human $\alpha 9 \alpha 10 \mathrm{nAChR}$ bound to RgIA4. (A,B) RgIA4 bound to the $\alpha 9(+) / \alpha 9(-)$ interface. (C,D) RgIA4 bound to the $\alpha 9(+) / \alpha 10(-)$ interface. (E,F) RgIA4 bound to the $\alpha 10(+) / \alpha 9(-)$ interface. Hydrogen bonds and salt bridges are represented by black dashed lines and red dashed lines, respectively.

At the $\alpha 10(+) / \alpha 9(-)$ binding interface (Figure 4E,F), interactions were very similar to the $\alpha 9(+) / \alpha 9(-)$ interface both on the principal side and complementary side. Like the $\alpha 9(+) / \alpha 9(-)$ binding interface, Arg7 of RgIA4 interacted with Tyr95 and Pro200 of $\alpha 10$ subunit through hydrogen bonds. Additionally, Tyr199 of the $\alpha 10$ subunit interacted 
with Arg11 of RgIA4 through hydrogen, which was not involved in other interfaces at the principal side. On the complementary side, IYR10 of rgIA4 also formed hydrogen bonds with Arg81 and Arg113 of the $\alpha 9$ subunit. Compared with the other two interfaces, additional hydrogen bonds occurred between Thr38 and Arg59 of the $\alpha 9$ subunit and CIR9 of RgIA4. This difference may explain the closed affinity gap of RgIA4 across the rodent and human $\alpha 9 \alpha 10$ nAChR.

Additionally, Tyr199 of $\alpha 10$ subunit interacted with Arg11 of RgIA4 through hydrogen, which was not involved in other interfaces at the principal side. On the complementary side, IYR10 of rgIA4 also formed hydrogen bonds with Arg81 and Arg113 of $\alpha 9$ subunit. Compared with the other two interfaces, additional hydrogen bonds occurred between Thr38 and Arg59 of $\alpha 9$ subunit and CIR9 of RgIA4. This difference may explain the closed affinity gap of RgIA4 across the rodent and human $\alpha 9 \alpha 10 \mathrm{nAChR}$.

\section{Discussion}

Conotoxin is a kind of peptide isolated from the venoms of marine cone snails that display potency and inherent selectivity at mammalian nAChRs. Based on their structure, function, and respective receptor target, conotoxins were divided into different classes. $\alpha$ conotoxin is a class of conotoxin, which usually act as competitive antagonists in potential mechanisms of pain. $\alpha 9 \alpha 10 \mathrm{nAChR}$ was implicated in pain and proposed to be a valid molecular target for pain-related drug development [24]. Early publications suggested that Vc1.1 and RgIA, two $\alpha$-conotoxins, were reported to display the potential pain-relieving actions in a rat model $[25,26]$. Subsequently, in silico studies and electrophysiological experiments have further elucidated the binding properties of Vc1.1 and RgIA at the $\alpha 9 \alpha 10$ $\mathrm{nAChR}[25,27]$.

Over the past decade, AChBPs, which showed a structural and functional homologue of ligand-binding domain of nAChRs, have been widely used as models for nAChRs [22]. Previously, there were many crystal structures of different $\alpha$-conotoxins complexed with AcAChBP that revealed the key residues in $\alpha$-conotoxins or nAChRs, namely for the GIC/AcAChBP complex [28], LvIA / Ac-AChBP complex [29], ImI/Ac-AChBP complex [30], and PnIA variant- $A c-A C h B P$ complex [31]. Moreover, there are also many conotoxin analogs that were designed based on the AChBP-conotoxin structures. These include [S9A]TxID and [S9K]TxID for $\alpha 3 \beta 4 \mathrm{nAChR}$ [32,33], [S4A,E11A,L15A]MII for $\alpha 6$-containing nAChRs [34], and [S4K,N9A]Vc1.1 for $\alpha 9 \alpha 10 \mathrm{nAChR}$ [35]. According to the considerable homology between $\mathrm{AChBP}$ and $\mathrm{nAChR}$, especially in their ligand-binding domain, exploring the interactions between $\alpha$-conotoxin and $\alpha 9 \alpha 10 \mathrm{nAChR}$ can be less difficult. Although Marios Zouridakis et al. reported the X-ray structure of the extracellular domain of $\alpha 9$ subunit in complex with RgIA, they only showed the principal side in the RgIA / $\alpha 9 \alpha 10 \mathrm{nAChR}$ interface. Here, we present two crystal structures of Ac-AChBP in complex with RgIA and RgIA4, respectively. It is the first time a complex between $A c-A C h B P$ and pain-related conotoxin was reported and the first time the complementary side in RgIA/RgIA4- $\alpha 9 \alpha 10 \mathrm{nAChR}$ interface through the homologous protein AChBP was shown.

Comparing RgIA/RgIA4-Ac-AChBP complexes with our previously reported conotoxinAChBP complexes, the conotoxin bound $A c-A C h B P$ showed similar conformation in its interface, even in loop C of Ac-AChBP (Figure S4A). All conotoxins between two adjacent AChBP subunits and its N-termini and C-termini were located at the bottom and top of the binding interface, respectively (Figure 2A,B). Among these $\alpha$-conotoxins, the $\mathrm{C}$-termini were superposed worse than those of N-termini. Through sequence alignment of GIC, LvIA, RgIA, and RgIA4, it is clearly shown that all conotoxin exhibited high homology in their N-termini (Figure S4B). However, the sequences in C-termini were significantly different. Thus, we think the sequence variations in C-termini may contribute to the unique selectivity of different $\mathrm{nAChRs}$ or determine its preferential receptor antagonism of $\alpha$-conotoxin.

RgIA was firstly reported as a potent and specific blocker of the $\alpha 9 \alpha 10 \mathrm{nAChR}$ [26]. Then, Romero HK et al. published an analog of RgIA, RgIA4, which displayed higher 
potency than RgIA for both human and rat $\alpha 9 \alpha 10 \mathrm{nAChR}$ [26]. In this study, we determined the crystal structure of the complex of $A c-A C h B P$ with RgIA and RgIA4, respectively, revealing the reason of the higher affinity and selective target of RgIA4. As shown in Figure 3, the local conformation of conotoxin changed a little when Arg9 and Tyr10 of RgIA were replaced with CIR9 and IYR10 of RgIA4, respectively. Whether in RgIA or RgIA4, it was obviously shown that Asp5, Arg7, and Arg11 play key roles in principal side. In the RgIA/Ac-AChBP complex, Arg9 and Tyr10 of RgIA interacted with Ac-AChBP mainly in complementary side. The marked shift was observed in position 9 and position 10 of conotoxins. In the complementary side of RgIA/Ac-AChBP interface, Arg9 of RgIA interacted with Gln55 and Met114 of Ac-AChBP through Van der Waal contact. When CIR9 was introduced into the conotoxin backbone, it formed hydrogen bonds with Thr34 and Gln55, respectively (Figure 3B,D and Table 1). Similar to Arg9 in RgIA, substitute Tyr10 of RgIA to IYR introduced additional interaction between IYR10 of RgIA4 and Arg77 of Ac-AChBP. Thus, the side chains change of Arg9 and Tyr10 of RgIA may compensate for the affinity increase of RgIA with the receptor, which explains the observation that RgIA4 produced a complete block of the nAChR with low nanomolar potencies [15].

Notably, the ligand-binding domains of $\alpha 9$ and $\alpha 10$ subunits had a remarkable sequence similarity with Ac-AChBP (Figure 2D). Given that RgIA and RgIA4 are specific antagonists to $\alpha 9 \alpha 10 \mathrm{nAChR}$, we performed MD simulations based on crystal structures of RgIA/Ac-AChBP and RgIA4/Ac-AChBP, respectively, to assess the possibility of conotoxins for any of these sites. Previously, RgIA was reported to show a favorable binding at $\alpha 9(+) / \alpha 9(-)$ or $\alpha 10(+) / \alpha 9(-)$ rather than the traditional $\alpha 9(+) / \alpha 10(-)$ interface based on the electrostatic potential distribution analysis [17]. Because RgIA4 is the analog of RgIA, the electrostatic distribution of RgIA4 may be similar to RgIA. Together with the previous research and our contact analysis, the $\alpha 9(+) / \alpha 10(-)$ interface in RgIA4- $\alpha 9 \alpha 10 \mathrm{nAChR}$ interaction is also undesired. Combined with sequence alignment and MD simulation, key residues of $A c-A C h B P, \alpha 9$ and $\alpha 10$ subunits in the principal side were highly conserved in nAChR subunits and Ac-AChBP, such as Tyr95, Trp151, Tyr192, Glu197, Tyr199, and Pro200 of $\alpha 9$ or $\alpha 10$ subunits. It is worth mentioning that the importance of the abovementioned conserved residues were mostly evaluated by mutational studies previously. Mutated Trp151 to threonine in the $\alpha 9$ subunit and their co-expression in oocytes was less potently inhibited by RgIA [13]. Additionally, the single mutation of $\alpha 10-$ Glu197 or $\alpha 10-$ Pro200 to glutarnine led to 25- and 300-fold decreased sensitivity to RgIA [36]. Additionally, when $\alpha 10$ subunits act as (+) side in RgIA/ $\alpha 9 \alpha 10$ nAChR complex, the important interaction between Arg7 of RgIA and Asp201 of $\alpha 10$ subunit cannot be ignored, though there are no functional analyses of Asp201. In contrast to principal side, most interacted residues in the complementary side of the RgIA/RgIA4- $\alpha 9 \alpha 10$ nAChR interface are significantly different with nAChR subunits or Ac-AChBP. The main interaction on this side are hydrogen bonds and the interacted residues involved here are poorly investigated. On this side, only Asp121 of the $\alpha 9$ subunit is previously demonstrated to have critical roles through mutation analyses [36]. Apart from Asp121, Thr38, Trp57, Arg58, Arg81, Asp168, Ser170, and Asp171 of $\alpha 9$ subunit also show their importance in binding RgIA/RgIA4 in our MD simulation in RgIA- $\alpha 9 \alpha 10 \mathrm{nAChR}$ complex and RgIA4- $\alpha 9 \alpha 10 \mathrm{nAChR}$ complex. Among these residues, Trp57, Arg81, Asp168 and Asp171 are highly conserved in most nAChR subunits, and S170 is unique in the $\alpha 9$ subunit. Compared with the model of the RgIA- $\alpha 9 \alpha 10$ nAChR complex with RgIA4/ $\alpha 9 \alpha 10$ nAChR complex, Ser79 and Arg113 of the $\alpha 9$ subunit are specifically exist in the model RgIA4/ $\alpha 9 \alpha 10 \mathrm{nAChR}$ complex. Interestingly, position 79 and 113 in the $\beta 2$ and $\beta 4$ subunits are previously reported to affect the sensitivity of receptor to $\alpha$-conotoxin RegIIA [37,38]. Thus, Ser79 and Arg113 of the $\alpha 9$ subunit may also be important in increasing the affinity to conotoxins.

Taken together, this is the first time the structure of the antagonist of $\alpha 9 \alpha 10 \mathrm{nAChR}$ with Ac-AChBP was shown. The structures show the key residues of RgIA/RgIA4 in binding Ac-AChBP. Based on the structures of the complex, we performed MD simulation and revealed the interactions between $\mathrm{RgIA} / \mathrm{RgIA} 4$ and $\alpha 9 \alpha 10 \mathrm{nAChR}$ in detail and proposed 
the important residues in the complementary side. These results may be valuable in the design and development of potent $\alpha 9 \alpha 10$-selective drugs, with significant implications for the treatment of neuropathic pain.

\section{Methods and Materials}

\subsection{Overall Structure of $\mathrm{RgIA}$ and $\mathrm{RgIA4}$ Bound to Ac-AChBP}

Linear peptides were assembled using solid-phase methodology on an ABI 433A peptide synthesizer (Applied Biosystems Inc., Foster City, CA, USA). Cys residues were protected in pairs with S-trityl (Trt) on CysI and CysIII (the first and third Cys) and S-acetamidomethyl (Acm) on the second and fourth Cys. The first disulfide bond (CysICysIII) was formed under an oxidative condition using $20 \mathrm{mM}$ potassium ferricyanide $\mathrm{K}_{3}\left[\mathrm{Fe}(\mathrm{CN})_{6}\right]$, and $0.1 \mathrm{M}$ Tris- $\mathrm{HCl}$, pH 7.5, which was reacted for $45 \mathrm{~min}$, and the monocyclic peptide was purified by reverse-phase HPLC. Then, the Acm groups were cleaved and the second disulfide bond (CysII-CysIV) was formed by iodine oxidation. The bicyclic peptide was purified by HPLC on a reversed-phase C18 Vydac column (Agilent Technologies, Hesperia, CA, USA) with a linear gradient of 10-40\% B90 in $30 \mathrm{~min}$. Solvent A was $0.1 \%$ TFA in $\mathrm{H}_{2} \mathrm{O}$, Solvent $\mathrm{B}$ include $90 \% \mathrm{CAN}$, and $0.092 \%$ TFA in $\mathrm{H}_{2} \mathrm{O}$. The purity of final product was confirmed by reversed phase-HPLC (Waters ACQUITY UPLC H-Class) and ESI-IT-TOF (Shimadzu, Tokyo, Japan) mass spectrometry.

\subsection{Protein Expression and Purification}

The Ac-AChBP was expressed using the Bac-to-Bac baculovirus system (Invitrogen). Ac-AChBP with an N-terminal gp67 signal peptide was used to facilitate secretion and a C-terminal six-histidine tag was cloned into the pFastBac Dual vector (Invitrogen) using ClonExpress II One Step Cloning Kit (Vazyme Biotech Co., Ltd., Nanjing, China). The construct was transformed into bacterial DH10Bac competent cells, and the extracted bacmid was then transfected into Sf9 cells in the presence of Cellfectin II Reagent (Invitrogen) to produce recombinant baculoviruses. After two cycles of amplification, the high-titer viruses were used to infect Hi5 insect cells with the density of $2 \times 10^{6}$ cells per ml in HyQ SFX medium (HyClone). The supernatant of cell culture containing Ac-AChBP was harvested $48 \mathrm{~h}$ after infection and purified with nickel-nitrilotriacetic acid resin (GE Healthcare) in HBS buffer (10 mM Hepes, pH 7.2, $150 \mathrm{mM} \mathrm{NaCl),} \mathrm{followed} \mathrm{by} \mathrm{gel-filtration} \mathrm{chromatogra-}$ phy using the Superdex 200 column (GE Healthcare, Chicago, IL, USA). Purified Ac-AChBP and synthetic conotoxins were mixed with a molar ratio of protein to conotoxin equal to $1: 2.5$, then left on ice overnight. Gel-filtration chromatography was utilized again to obtain the conotoxin/Ac-AChBP complex.

\subsection{Crystallization, Data Collection and Structural Determination}

The purified $\alpha$-CTx/Ac-AChBP complex was concentrated to $\sim 20 \mathrm{mg} / \mathrm{mL}$ for crystallization. The crystals were grown at $18^{\circ} \mathrm{C}$ by mixing an equal volume of protein and reservoir solutions using the sitting drop vapor diffusion method. For crystal growth of the RgIA/Ac-AChBP complex, the reservoir solution contained 1.8 M magnesium sulfate hydrate and 0.1 M sodium acetate trihydrate $\mathrm{pH}$ 4.6. For the RgIA4/Ac-AChBP complex, the reservoir solution contained $1.8 \mathrm{M}$ sodium phosphate monobasic monohydrate and potassium phosphate dibasic, pH5.0. All crystals were cryoprotected in well solution supplemented with $20 \%$ (vol/vol) glycol and were cooled to liquid-nitrogen before data collection. All diffraction data were collected on the BL17U1 beamline at Shanghai Synchrotron Research Facility (SSRF) [39] and processed with HKL2000 [40]. The structures of $\alpha-\mathrm{CTx} / \mathrm{Ac}$-AChBP complexes were determined by the molecular replacement method with the crystallographic program PHASER in the CCP4 suite [41]. The program PHENIX and COOT were used to iterative refinement of all structures [42,43]. Structural validations were performed with the program MolProbity [44]. All structural figures used here were generated with PYMOL (http: / / www.pymol.org/, accessed on 4 November 2021). All 
diffraction data collection, processing, and structural refinement statistics are listed in Table S1.

\subsection{Homology Modeling and Model Refinement}

The homology models of the extracellular ligand-binding domain of human $\alpha 9 \alpha 10$ nAChR bound to RgIA4 and RgIA were generated using the Swiss-Model. The crystal structures of $A c-\mathrm{AChBP}$ in complex with RgIA4/RgIA were used as templates to model the the RgIA/RgIA4-bound $\alpha 9 \alpha 10 \mathrm{nAChR}$. Subsequently, generated models were checked by PDBsum, which includes a full PROCHECK assessment of each protein's geometry. For model refinement, force field ff14SB was used. For noncanonical residues, force field library and parameters were generated by R.E.D. Server Development $[45,46]$. In this pipeline, partial charges are calculated by constructing $\mathrm{ACE} / \mathrm{NME}(\mathrm{ACE}=\mathrm{CH} 3 \mathrm{CO}$ and $\mathrm{NME}=\mathrm{NHCH} 3$ ) capped amino acid (the central fragment is new amino acid). By adding two intra-molecular charge constraints (equal 0), we could acquire central fragment atom charges and then the force field library. When setting up molecular dynamic simulation, we used the GPU-accelerated software AMBER v18 and introduced the ff14SB force field and the new force field together $[47,48]$. The complexes were solvated in the SPCE water box. Sodium ions were added to neutralize the systems. The systems were minimized with 1000 steps twice and the first minimization was performed with the solute restrained to their position by a harmonic force of $500 \mathrm{kcal} / \mathrm{mol} \cdot \AA^{2}$. The second minimization was then performed with all position restraints withdrawn. The systems were then gradually heated up from 0 to $1000 \mathrm{~K}$ over $40 \mathrm{ps}$ with restrained to the position of potential non-interaction residues using a $500 \mathrm{kcal} / \mathrm{mol} \cdot \AA^{2}$ force potential and then gradually cooled down to $0 \mathrm{~K}$ over $120 \mathrm{ps}$ with the same restraint. The MD-simulation of these two processes used a time step of $1 \mathrm{fs}$. Another minimization was conducted without position restraint through 500 steps.

Supplementary Materials: The following is available online at https://www.mdpi.com/article/ 10.3390/md19120709/s1. Figure S1: The HPLC and mass spectra profiles of RgIA/RgIA4; Figure S2: Ramachandran plot of the homology models of two possible stoichiometries $(\alpha 9)_{2}(\alpha 10)_{3}$ and $(\alpha 9)_{3}(\alpha 10)_{2}$; Figure S3: The molecular dynamics models of human $\alpha 9 \alpha 10 \mathrm{nAChR}$ bound to RgIA; Figure S4: Comparison of different $\alpha$-CTxs bound by Ac-AChBP; Table S1: The relevant data collection and refinement statistics of two $\mathrm{X}$-ray crystal structures.

Author Contributions: S.P. and Y.F. contributed equally to this work. S.L., X.W., S.P. and Y.F. conceived and designed the study. S.P. expressed, purified, and crystallized the protein. S.P., Y.F. and Y.X. performed computational modeling. X.Z. conducted peptide synthesis. S.P., Y.F., S.L. and X.W. analyzed the data and wrote the paper. All authors have read and agreed to the published version of the manuscript.

Funding: This work was founded by High Level Introduction of Talent Research Start-up Fund of Putian University, 2020009.

Acknowledgments: We are grateful to the staff scientists at the BL17U beamline of the Shanghai Synchrotron Research Facility (SSRF) and the X-ray crystallography platform of the Tsinghua University Technology Center for Protein Research for assistance with data collection and processing. We thank Igor E. Kasheverov and Victor I. Tsetlin (Shemyakin-Ovchinnikov Institute of Bioorganic Chemistry, Russian Academy of Sciences, Moscow, Russia) for providing the plasmid of Ac-AChBP.

Conflicts of Interest: The authors declare no conflict of interest.

\section{References}

1. Miller, P.S.; Smart, T.G. Binding, activation and modulation of Cys-loop receptors. Trends Pharmacol. Sci. 2010, 31, 161-174. [CrossRef] [PubMed]

2. Davies, P.A.; Wang, W.; Hales, T.G.; Kirkness, E.F. A novel class of ligand-gated ion channel is activated by $\mathrm{Zn}^{2+}$. J. Biol. Chem. 2003, 278, 712-717. [CrossRef] [PubMed]

3. Gotti, C.; Clementi, F. Neuronal nicotinic receptors: From structure to pathology. Prog. Neurobiol. 2004, 74, 363-396. [CrossRef] [PubMed] 
4. Gotti, C.; Clementi, F.; Fornari, A.; Gaimarri, A.; Guiducci, S.; Manfredi, I.; Moretti, M.; Pedrazzi, P.; Pucci, L.; Zoli, M. Structural and functional diversity of native brain neuronal nicotinic receptors. Biochem. Pharmacol. 2009, 78, 703-711. [CrossRef]

5. Dellisanti, C.D.; Ghosh, B.; Hanson, S.M.; Raspanti, J.M.; Grant, V.A.; Diarra, G.M.; Schuh, A.M.; Satyshur, K.; Klug, C.S.; Czajkowski, C. Site-directed spin labeling reveals pentameric ligand-gated ion channel gating motions. PLoS Biol. 2013, 11, e1001714. [CrossRef]

6. Dineley, K.T.; Pandya, A.A.; Yakel, J.L. Nicotinic ACh receptors as therapeutic targets in CNS disorders. Trends Pharmacol. Sci. 2015, 36, 96-108. [CrossRef]

7. Terlau, H.; Olivera, B.M. Conus venoms: A rich source of novel ion channel-targeted peptides. Physiol. Rev. 2004, 84, 41-68. [CrossRef]

8. Mohammadi, S.A.; Christie, M.J. Conotoxin interactions with $\alpha 9 \alpha 10$-nAChRs: Is the $\alpha 9 \alpha 10$-nicotinic acetylcholine receptor an important therapeutic target for pain management? Toxins 2015, 7, 3916-3932. [CrossRef]

9. Castro, J.; Harrington, A.M.; Garcia-Caraballo, S.; Maddern, J.; Grundy, L.; Zhang, J.; Page, G.; Miller, P.E.; Craik, D.J.; Adams, D.J. $\alpha$-Conotoxin Vc1. 1 inhibits human dorsal root ganglion neuroexcitability and mouse colonic nociception via GABAB receptors. Gut 2017, 66, 1083-1094. [CrossRef]

10. Hone, A.J.; McIntosh, J.M. Nicotinic acetylcholine receptors in neuropathic and inflammatory pain. FEBS Lett. 2018, 592, 1045-1062. [CrossRef]

11. Sgard, F.; Charpantier, E.; Bertrand, S.; Walker, N.; Caput, D.; Graham, D.; Bertrand, D.; Besnard, F. A novel human nicotinic receptor subunit, alpha10, that confers functionality to the alpha9-subunit. Mol. Pharmacol. 2002, 61, 150-159. [CrossRef]

12. Elgoyhen, A.B.; Vetter, D.E.; Katz, E.; Rothlin, C.V.; Heinemann, S.F.; Boulter, J. alpha10: A determinant of nicotinic cholinergic receptor function in mammalian vestibular and cochlear mechanosensory hair cells. Proc. Natl. Acad. Sci. USA 2001, 98, 3501-3506. [CrossRef]

13. Ellison, M.; Feng, Z.P.; Park, A.J.; Zhang, X.; Olivera, B.M.; McIntosh, J.M.; Norton, R.S. Alpha-RgIA, a novel conotoxin that blocks the alpha9alpha10 nAChR: Structure and identification of key receptor-binding residues. J. Mol. Biol. 2008, 377, 1216-1227. [CrossRef]

14. Azam, L.; McIntosh, J.M. Molecular basis for the differential sensitivity of rat and human alpha9alpha10 nAChRs to alphaconotoxin RgIA. J. Neurochem. 2012, 122, 1137-1144. [CrossRef]

15. Romero, H.K.; Christensen, S.B.; Di Cesare Mannelli, L.; Gajewiak, J.; Ramachandra, R.; Elmslie, K.S.; Vetter, D.E.; Ghelardini, C.; Iadonato, S.P.; Mercado, J.L.; et al. Inhibition of alpha9alpha10 nicotinic acetylcholine receptors prevents chemotherapy-induced neuropathic pain. Proc. Natl. Acad. Sci. USA 2017, 114, E1825-E1832. [CrossRef]

16. Christensen, S.B.; Hone, A.J.; Roux, I.; Kniazeff, J.; Pin, J.P.; Upert, G.; Servent, D.; Glowatzki, E.; McIntosh, J.M. RgIA4 Potently Blocks Mouse alpha9alpha10 nAChRs and Provides Long Lasting Protection against Oxaliplatin-Induced Cold Allodynia. Front. Cell Neurosci. 2017, 11, 219. [CrossRef]

17. Zouridakis, M.; Papakyriakou, A.; Ivanov, I.A.; Kasheverov, I.E.; Tsetlin, V.; Tzartos, S.; Giastas, P. Crystal Structure of the Monomeric Extracellular Domain of alpha9 Nicotinic Receptor Subunit in Complex With alpha-Conotoxin RgIA: Molecular Dynamics Insights Into RgIA Binding to alpha9alpha10 Nicotinic Receptors. Front. Pharmacol. 2019, 10, 474. [CrossRef]

18. Unwin, N. Refined structure of the nicotinic acetylcholine receptor at 4A resolution. J. Mol. Biol. 2005, 346, 967-989. [CrossRef]

19. Li, S.X.; Huang, S.; Bren, N.; Noridomi, K.; Dellisanti, C.D.; Sine, S.M.; Chen, L. Ligand-binding domain of an alpha7-nicotinic receptor chimera and its complex with agonist. Nat. Neurosci. 2011, 14, 1253-1259. [CrossRef]

20. Morales-Perez, C.L.; Noviello, C.M.; Hibbs, R.E. X-ray structure of the human $\alpha 4 \beta 2$ nicotinic receptor. Nature 2016, 538, 411-415. [CrossRef]

21. Kouvatsos, N.; Giastas, P.; Chroni-Tzartou, D.; Poulopoulou, C.; Tzartos, S.J. Crystal structure of a human neuronal nAChR extracellular domain in pentameric assembly: Ligand-bound $\alpha 2$ homopentamer. Proc. Natl. Acad. Sci. USA 2016, 113, 9635-9640. [CrossRef]

22. Brejc, K.; van Dijk, W.J.; Klaassen, R.V.; Schuurmans, M.; van Der Oost, J.; Smit, A.B.; Sixma, T.K. Crystal structure of an ACh-binding protein reveals the ligand-binding domain of nicotinic receptors. Nature 2001, 411, 269-276. [CrossRef]

23. Smit, A.B.; Syed, N.I.; Schaap, D.; van Minnen, J.; Klumperman, J.; Kits, K.S.; Lodder, H.; van der Schors, R.C.; van Elk, R.; Sorgedrager, B.; et al. A glia-derived acetylcholine-binding protein that modulates synaptic transmission. Nature 2001, 411, 261-268. [CrossRef]

24. Vazquez, A.E. $\alpha 9 \alpha 10$ Acetylcholine receptors: Structure and functions. Neurotransmitter 2016, 3, e1298.

25. Vincler, M.; Wittenauer, S.; Parker, R.; Ellison, M.; Olivera, B.M.; McIntosh, J.M. Molecular mechanism for analgesia involving specific antagonism of alpha9alpha10 nicotinic acetylcholine receptors. Proc. Natl. Acad. Sci. USA 2006, 103, 17880-17884. [CrossRef]

26. Ellison, M.; Haberlandt, C.; Gomez-Casati, M.E.; Watkins, M.; Elgoyhen, A.B.; McIntosh, J.M.; Olivera, B.M. Alpha-RgIA: A novel conotoxin that specifically and potently blocks the alpha9alpha10 nAChR. Biochemistry 2006, 45, 1511-1517. [CrossRef]

27. Perez, E.G.; Cassels, B.K.; Zapata-Torres, G. Molecular modeling of the alpha9alpha10 nicotinic acetylcholine receptor subtype. Bioorg. Med. Chem. Lett. 2009, 19, 251-254. [CrossRef]

28. Lin, B.; Xu, M.; Zhu, X.; Wu, Y.; Liu, X.; Zhangsun, D.; Hu, Y.; Xiang, S.-H.; Kasheverov, I.E.; Tsetlin, V.I.; et al. From crystal structure of $\alpha$-conotoxin GIC in complex with Ac-AChBP to molecular determinants of its high selectivity for $\alpha 3 \beta 2 \mathrm{nAChR}$. Sci. Rep. 2016, 6, 22349. [CrossRef] 
29. Xu, M.; Zhu, X.; Yu, J.; Yu, J.; Luo, S.; Wang, X. The crystal structure of Ac-AChBP in complex with $\alpha$-conotoxin LvIA reveals the mechanism of its selectivity towards different nAChR subtypes. Protein Cell 2017, 8, 675-685. [CrossRef]

30. Hansen, S.B.; Sulzenbacher, G.; Huxford, T.; Marchot, P.; Taylor, P.; Bourne, Y. Structures of Aplysia AChBP complexes with nicotinic agonists and antagonists reveal distinctive binding interfaces and conformations. EMBO J. 2005, 24, 3635-3646. [CrossRef]

31. Celie, P.H.; Kasheverov, I.E.; Mordvintsev, D.Y.; Hogg, R.C.; van Nierop, P.; van Elk, R.; van Rossum-Fikkert, S.E.; Zhmak, M.N.; Bertrand, D.; Tsetlin, V.; et al. Crystal structure of nicotinic acetylcholine receptor homolog AChBP in complex with an alpha-conotoxin PnIA variant. Nat. Struct. Mol. Biol. 2005, 12, 582-588. [CrossRef] [PubMed]

32. Wu, Y.; Zhangsun, D.; Zhu, X.; Kaas, Q.; Zhangsun, M.; Harvey, P.J.; Craik, D.J.; McIntosh, J.M.; Luo, S. alpha-Conotoxin [S9A]TxID Potently Discriminates between alpha3beta4 and alpha6/alpha3beta4 Nicotinic Acetylcholine Receptors. J. Med. Chem. 2017, 60, 5826-5833. [CrossRef] [PubMed]

33. Yu, J.; Zhu, X.; Harvey, P.J.; Kaas, Q.; Zhangsun, D.; Craik, D.J.; Luo, S. Single Amino Acid Substitution in alpha-Conotoxin TxID Reveals a Specific alpha3beta4 Nicotinic Acetylcholine Receptor Antagonist. J. Med. Chem. 2018, 61, 9256-9265. [CrossRef] [PubMed]

34. Azam, L.; Yoshikami, D.; McIntosh, J.M. Amino acid residues that confer high selectivity of the alpha6 nicotinic acetylcholine receptor subunit to alpha-conotoxin MII[S4A,E11A,L15A]. J. Biol. Chem. 2008, 283, 11625-11632. [CrossRef] [PubMed]

35. Halai, R.; Clark, R.J.; Nevin, S.T.; Jensen, J.E.; Adams, D.J.; Craik, D.J. Scanning mutagenesis of alpha-conotoxin Vc1.1 reveals residues crucial for activity at the alpha9alpha10 nicotinic acetylcholine receptor. J. Biol. Chem. 2009, 284, 20275-20284. [CrossRef] [PubMed]

36. Azam, L.; Papakyriakou, A.; Zouridakis, M.; Giastas, P.; Tzartos, S.J.; McIntosh, J.M. Molecular interaction of alpha-conotoxin RgIA with the rat alpha9alpha10 nicotinic acetylcholine receptor. Mol. Pharmacol. 2015, 87, 855-864. [CrossRef] [PubMed]

37. Cuny, H.; Kompella, S.N.; Tae, H.S.; Yu, R.; Adams, D.J. Key Structural Determinants in the Agonist Binding Loops of Human beta2 and beta4 Nicotinic Acetylcholine Receptor Subunits Contribute to alpha3beta4 Subtype Selectivity of alpha-Conotoxins. J. Biol. Chem. 2016, 291, 23779-23792. [CrossRef] [PubMed]

38. Kompella, S.N.; Hung, A.; Clark, R.J.; Mari, F.; Adams, D.J. Alanine scan of alpha-conotoxin RegIIA reveals a selective alpha3beta4 nicotinic acetylcholine receptor antagonist. J. Biol. Chem. 2015, 290, 1039-1048. [CrossRef] [PubMed]

39. Wang, Q.-S.; Zhang, K.-H.; Cui, Y.; Wang, Z.-J.; Pan, Q.-Y.; Liu, K.; Sun, B.; Zhou, H.; Li, M.-J.; Xu, Q.; et al. Upgrade of macromolecular crystallography beamline BL17U1 at SSRF. Nucl. Sci. Tech. 2018, 29, 68. [CrossRef]

40. Otwinowski, Z.; Minor, W. Processing of X-ray diffraction data collected in oscillation mode. Methods Enzym. 1997, 276, 307-326.

41. McCoy, A.J.; Grosse-Kunstleve, R.W.; Adams, P.D.; Winn, M.D.; Storoni, L.C.; Read, R.J. Phaser crystallographic software. J. Appl. Crystallogr. 2007, 40, 658-674. [CrossRef]

42. Adams, P.D.; Grosse-Kunstleve, R.W.; Hung, L.W.; Ioerger, T.R.; McCoy, A.J.; Moriarty, N.W.; Read, R.J.; Sacchettini, J.C.; Sauter, N.K.; Terwilliger, T.C. PHENIX: Building new software for automated crystallographic structure determination. Acta Cryst. D Biol. Cryst. 2002, 58, 1948-1954. [CrossRef]

43. Emsley, P.; Cowtan, K. Coot: Model-building tools for molecular graphics. Acta Cryst. D Biol. Cryst. 2004, 60, $2126-2132$. [CrossRef]

44. Chen, V.B.; Arendall, W.B., 3rd; Headd, J.J.; Keedy, D.A.; Immormino, R.M.; Kapral, G.J.; Murray, L.W.; Richardson, J.S.; Richardson, D.C. MolProbity: All-atom structure validation for macromolecular crystallography. Acta Cryst. D Biol. Cryst. 2010, 66, 12-21. [CrossRef]

45. Vanquelef, E.; Simon, S.; Marquant, G.; Garcia, E.; Klimerak, G.; Delepine, J.C.; Cieplak, P.; Dupradeau, F.Y. RED Server: A web service for deriving RESP and ESP charges and building force field libraries for new molecules and molecular fragments. Nucleic Acids Res. 2011, 39, W511-W517. [CrossRef]

46. Dupradeau, F.Y.; Pigache, A.; Zaffran, T.; Savineau, C.; Lelong, R.; Grivel, N.; Lelong, D.; Rosanski, W.; Cieplak, P. The R.E.D. tools: Advances in RESP and ESP charge derivation and force field library building. Phys. Chem. Chem. Phys. 2010, 12, 7821-7839. [CrossRef]

47. Salomon-Ferrer, R.; Gotz, A.W.; Poole, D.; Le Grand, S.; Walker, R.C. Routine Microsecond Molecular Dynamics Simulations with AMBER on GPUs. 2. Explicit Solvent Particle Mesh Ewald. J. Chem. Theory Comput. 2013, 9, 3878-3888. [CrossRef]

48. Maier, J.A.; Martinez, C.; Kasavajhala, K.; Wickstrom, L.; Hauser, K.E.; Simmerling, C. ff14SB: Improving the Accuracy of Protein Side Chain and Backbone Parameters from ff99SB. J. Chem. Theory Comput. 2015, 11, 3696-3713. [CrossRef] 